\title{
An inter-FPGA communication bus with error detection and dynamic clock phase adjustment
}

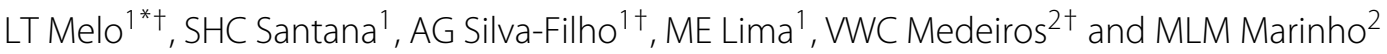

\begin{abstract}
Background: Nowadays, systems involving multiple FPGAs are used for various scientific applications. Such systems require a data bus dedicated to the communication between FPGAs, which could be done through a LVDS type. Another important factor is that the routing that interconnects the LVDS pins on the platform should be precisely developed to avoid instabilities in communication. Unfortunately, many platforms available in the market do not observe such restrictions, limiting the throughput of the bus.

Methods: This paper presents an inter-FPGAs communication channel based on a DDR interface directed to this kind of platform. This approach promotes a stable communication between these devices without the use of LVDS pins. An error detection module was also designed to ensure the sending integrity and correct any errors on the bus. A mechanism for dynamic and automatic clock phase adjustment used on the bus was also implemented to ensure that the developed modules were compatible with other platforms.
\end{abstract}

Results: The channel has been implemented in a PROCStarlll platform and rates of 4.76 Gbps were achieved.

Conclusions: The channel has been validated on a commercial platform with success and the synthesis results, as well as the performance results obtained by using it in a real implementation of the RTM algorithm, are also presented.

Keywords: LVDS; FPGA; CRC; Communication inter-FPGAs

\section{Background}

Platforms that involve multiple field-programmable gate arrays (FPGAs) have been the target of several study fields such as prototyping of MPSoCs (multiprocessor system-on-chip), acceleration, and encryption algorithms $[1,2]$. For these systems to work efficiently using existing resources in FPGAs, an efficient communication must exist between the FPGAs available on the platform. This type of communication in next-generation FPGAs is usually established through type interfaces low-voltage differential signaling (LVDS) [3]. This type of signaling allows signal sending at high speed through a differential pair of parallel wire. The use of this feature enables data

*Correspondence: Itm3@cin.ufpe.br

tEqual contributor

${ }^{1}$ Center for Informatics, Federal University of Pernambuco, Av. Jornalista

Anibal Fernandes, s/n Cidade Universitaria, 50.740-560 Recife, Brazil

Full list of author information is available at the end of the article transmission between devices to be performed more efficiently, allowing a more secure communication when facing electromagnetic interference $[4,5]$. This configuration enables busses to achieve transfer rates of about $10 \mathrm{Gbps}$ by using more advanced devices such as the Xilinx Virtex [6] family of FPGAs, Altera Stratix V [7] FPGAs.

Currently, many FPGAs support LVDS interfaces, and by being properly allocated on the platform, they can provide data communication at a high transmission rate by using their transceivers. However, some platforms available in the market were not designed to accommodate the LVDS FPGA pin resources in their communication lines, thus preventing the LVDS transceiver use and therefore hindering the implementation of communication channels with high performance. Other factors such as distance between tracks, resistance, and capacitance balance are important and need to be observed by this type

\section{Springer}

(C) 2015 Melo et al. Open Access This article is distributed under the terms of the Creative Commons Attribution 4.0 International License (http://creativecommons.org/licenses/by/4.0/), which permits unrestricted use, distribution, and reproduction in any medium, provided you give appropriate credit to the original author(s) and the source, provide a link to the Creative Commons license, and indicate if changes were made. 
of platform manufacturers. Data transmission performed without these resources can lead to errors in data transfer when the transmission rate is high, as there is no guarantee that data integrity is maintained. This is because of all FPGA I/O (Input/Output) pins that are generally subject to physical interference. Due to this possible low performance in data transmission, systems using these resources will have to reduce their logic module speed to suit the channel and thereby reduce the incidence of communication error.

This paper presents a bi-directional synchronized communication channel (full-duplex) regardless of LVDS interface use. To ensure a good transmission rate of the transmitter, it is based on a double data rate (DDR) interface, and to ensure data integrity, detection modules based on the generation and error checking cyclic redundancy check (CRC) were developed. Additionally, a module to fit automatically and dynamically the clock phase of the communication bus was also created. An example using the bus for reverse time migration (RTM) processing algorithm in a multi-FPGA platform is also presented.

After the introduction section, this paper is structured as follows: The section "Related work" discusses the work related to this article. In the section "GiDEL PROCStarIII platform", the platform used in the experiments is shown. In the section "Methods", the developed architecture is presented. In the section "Results", the results of the experiments are shown. Finally, the section "Conclusions" contains the conclusion of this work.

\section{Related work}

In this section, some studies focusing on inter-FPGA communication are presented. The paper [8] presents an architecture for this purpose, and the work [9] proposes a solution for optimizing communication between different FPGA devices.

The work [8] presents an architecture for inter-FPGA full-duplex communication with 18 LVDS transmission tracks. The architecture is based on DDR interface and consists of a transmitter and a receiver. The authors demonstrate that it is possible to reach transfer rates of about 10 Gbps by using this approach. The architecture is designed to be implemented in a high-performance cluster (PARAMNet-3) [10]. The architecture comprises a network switch allowing package routing to be performed at high speed. Each board being part of the switch consists of four FPGAs from Xilinx Virtex-4 and transceivers (multi-gigabit transceivers) available inside each FPGA. These transceivers are used for communication with the other system boards.

The transmitter consists of a control unit, LVDS I/O buffers, and a serializer/deserializer (SERDES) module. These last two features are I/O already available in the Xilinx Virtex-4 FPGA. The SERDES module is a serializer/deserializer that receives the application data in parallel and serializes it. The LVDS output buffers receive this serialized data and implement the output to 18 tracks of differential pairs. Each track has a transfer rate of $625 \mathrm{Mbps}$, summing up a total rate of about $10 \mathrm{Gbps}$.

The receiver has its clock generated by the transmitter. This allows synchronization of both sides. It consists of a control module, delays, SERDES I/O buffers, and LVDS module. The delay modules are responsible for introducing a 5-10 ps jitter on the clock input from the transmitter. This strategy respects the valid data window and is necessary to ensure that signals are received in the receiver correctly. The clock used in the receiver is of $312.5 \mathrm{MHz}$ and is synchronized with the transmitter.

Communication error detection is performed on the network layer of the switch, and the authors have developed a test engine to generate errors in the communication channel. This engine is responsible for testing each LVDS track, and two instances were implemented in each FPGA. The testing environment consists of a PowerPC processor executing an application that communicates with the architecture via a UART interface. It can be configured with test parameters and statistics view on the application.

In [9], a solution for optimizing inter-FPGA communication using channel adaptation is shown. The work demonstrates that each platform has its own characteristics, and to migrate up the project to another platform, you often need to change the architecture to adapt to necessary changes. The communication bus width, pointto-point broadcast, or LVDS are some of the options that could be parameterized by abstracting from the users that need to change theirs. In that sense, the authors of the work propose a definable channel and one that is able to self-adapt the target architecture.

Initially, it seems to be an architecture that consists of a base control module, FIFOs (First In, First Out), and PHY modules transmit (TX) and receive (RX). The architecture supports SDR/DDR interfaces and point-to-point transmission or LVDS. It can therefore be used for SERDES modules, serialization/deserialization of data, and it can be defined whether the communication is unidirectional or bidirectional. Finally, the architecture allows the bus width to be configurable.

The channel adaptation, called chAdapt, consists of a hardware module that self-configures the transmission according to the platform characteristics. For this, a transmission of a bit sequence occurs repeatedly between the transmitter and receiver, initially to identify possible delays in the communication form. At that time, all the controls are optimized and clock alignment is performed by dynamically adjusting its phase.

The architecture has been tested in different FPGAs by using the RAPTOR [11] module platform as base. This 
platform allows the adaptation of different reconfigurable modules that consist of Xilinx FPGAs. The tests were performed with the following FPGAs: Spartan-3A, Spartan-6, Virtex-4, and Virtex-5. Several combinations were analyzed in order to find possible optimizations in the communication. As a result, the authors demonstrate a comparative table between combinations used, highlighting the relative optimization after the addition of chAdapt module architecture. The tests performed with the communication between Virtex-4 and Spartan-6 are noteworthy. A significant improvement in communication was observed after the introduction of chAdapt in these scenarios. The results indicate that although it was not possible to align the bus width for these tests, the channel offers a great way when implemented for the communication link validation and identifying best parameters for data transfer.

\section{GiDEL PROCStarlII platform}

The communication channel presented in this work was implemented in a GiDEL PROCStarIII platform (Fig. 1) [12]. This platform consists of four Altera Stratix III 260E and has three memory banks: one of 512 MB DDR2 memory and two banks composed of 8 DDR2 SODIMMs with up to $4 \mathrm{~GB}$ each.

Although the platform has four FPGAs, it is not possible for the inter-FPGA communication to use differential pairs available in these devices. This occurs because the FPGA pins used in the platform, directly connected to the bus, are $\mathrm{I} / \mathrm{O}$ common pins. Another aspect is that there are no guarantees related to the printed circuit board (PCB) layout of the platform. Characteristics like resistance, capacitance, and bus route length that connect the FPGAs are not guaranteed to obtain a stable communication. For those reasons, the high-speed communication cannot be achieved. The local bus is responsible for connecting all FPGAs to the host controller through PCIex8. The communication protocol for this bus is automatically generated in the ProcWizard [13] tool. The main bus connects all the FPGAs and has a width of 40 bits. Figure 2 shows the internal architecture of the PROCStarIII platform.

For data communication, two busses are available: VLRL bus and LR bus. Each bus connects the adjacent FPGAs available on the platform. The VL-RL bus has a width of 10 bits and is type $\mathrm{I} / \mathrm{O}$ common pins. The signals used in this bus have a voltage of $1.8 \mathrm{~V}$. The LR bus has a width of 100 bits and is also type $\mathrm{I} / \mathrm{O}$ common pins. The LR bus, besides being the largest available, concentrates a single bank of pins that can be used in the communication channel. This minimizes the impact related to data skew since their paths are aligned. Thus, potential signal delay problems are decreased. Due to these characteristics, the proposed architecture in this paper uses the LR bus to implement the communication channel.

The platform also offers JTAG connectors that can be used for debugging and has PSDB connectors (PROCStarIII daughterboards), which are high-speed interfaces used to connect other cards provided by the manufacturer (camera links, ethernet interfaces, etc.).

\section{Methods}

The communication system proposed by this work is defined as a parallel communication channel, synchronized and 32 bits wide. Each controller is comprised of two main modules: a transmitter and a receiver. A common clock synchronizes the communication in the channel. This system can then be coupled in each FPGA to allow data communication between these devices on the platform.

The entire system was designed to facilitate its adaptation to different projects. To make this possible, a FIFO input on the transmitter and a FIFO output on the receiver were used, making the design transparent to the user. The adjustments for each application should be made through few adjustments in the input and output parameters of each FIFO.

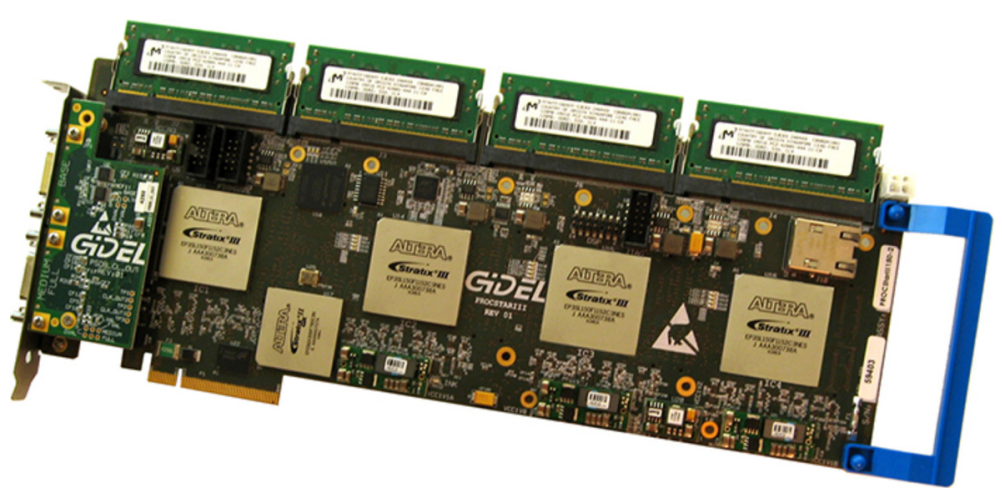

Fig. 1 GiDEL PROCStarlll platform. Photo of the platform used on the implementation 


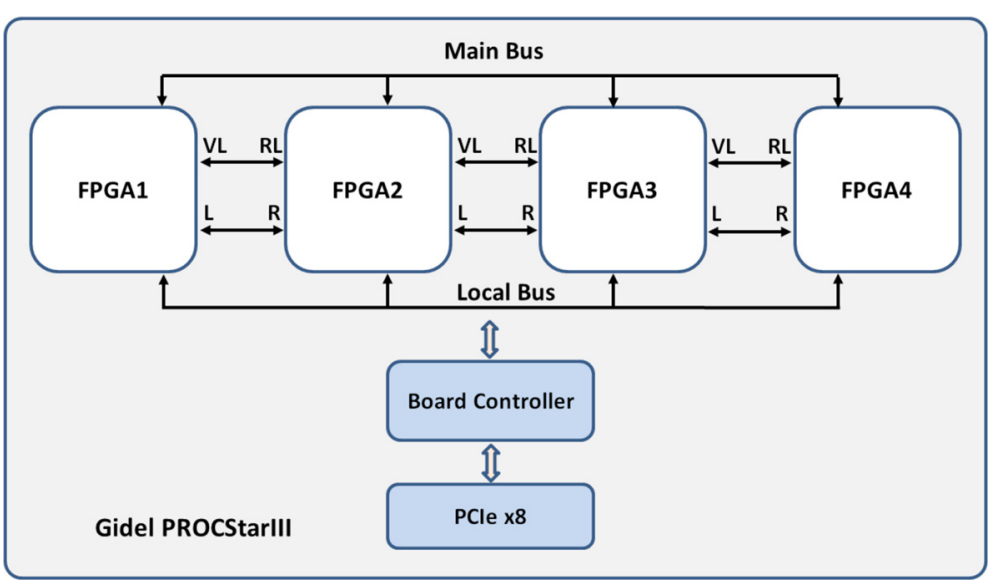

Fig. 2 GiDEL PROCStarlll platform diagrams. Overview of the platform architecture

Figure 3 shows the communication channel between two FPGAs involving the main signals from the transmitter and receiver.

One signal of 33 bits (data_bus) was defined for transferring data between devices. One bit of data_bus signal is intended for a valid signal, which indicates to the receiver that the data sent is valid and that it can be received and forwarded to the CRC check module and later to the FIFO output. Thus, the communication channel enables a data transfer with a width of 32 bits between the FPGAs.

The critical clk_bus signal is used to feed the clock signal to the receiver. The clock signal is generated in the transmitter through a PLL (Phase-Locked Loop) and sent to the receiver through a path reserved for this purpose. This ensures that both the transmitter and receiver are synchronized allowing the communication channel to operate stably. The synchronization and transmission failure signals that also incorporate the bus will be discussed in the next sections.

\section{Transmitter}

The transmission module, called Transmitter, is responsible for sending the data correctly through the bus. It is also its responsibility to align the clock with the availability of data on the bus, thus increasing utilization of the

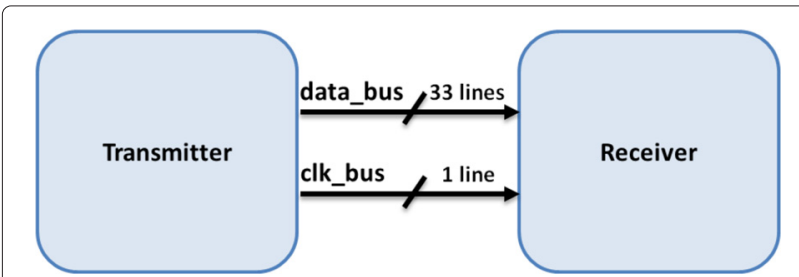

Fig. 3 Main signals from the communication bus. Detailed signalization of the communication bus data valid window, and to re-transmit the data if an error occurs in transmission. The transmitter also has a module for generating CRC for the data sent. After transmitting a data package, a checksum that validates the package is sent through the bus to be checked at the receiver. If errors are found during the verification, a request for retransmission is performed.

To perform these tasks, the Transmitter module is divided into five modules: a FIFO input (FIFO_INPUT), the retransmission circuit, the CRC generator $\left(C R C_{-}\right.$ $G E N)$, the physical layer (TRANS_PHY), and the control transmitter (TRANS_CTRL), as shown in Fig. 4.

The module FIFO_INPUT is available in MegaWizard that goes along with the tool development Altera Quartus II. This input module aims to standardize the interface of the transmission module with the architecture in which it is inserted and receive the data to be transferred. Its architecture allows it to work internally with a different frequency from the transmitter module to which it is coupled. Commonly, its internal frequency is higher than the one used in the system, and thus, the speed of data removal from the FIFO may be greater than the speed at which it is inserted.

For greater flexibility in the transmission process, the data width that will be inserted and FIFO depth are adjustable as needed through the use of configuration parameters of the transmitter. By default, the data width is of 128 bits and the FIFO depth is of 256 words.

Some signals are provided by FIFO input and should be used by the system to manipulate the data written in the transmitter input. The $w r_{-} c l k$ signal corresponds to the clock system that is used to control the data writing clock that will be forwarded to the communication channel. As previously mentioned, this clock is independent from the internal clock. The wr_req signal is used to indicate that the data entry wr_data is valid and should be written 


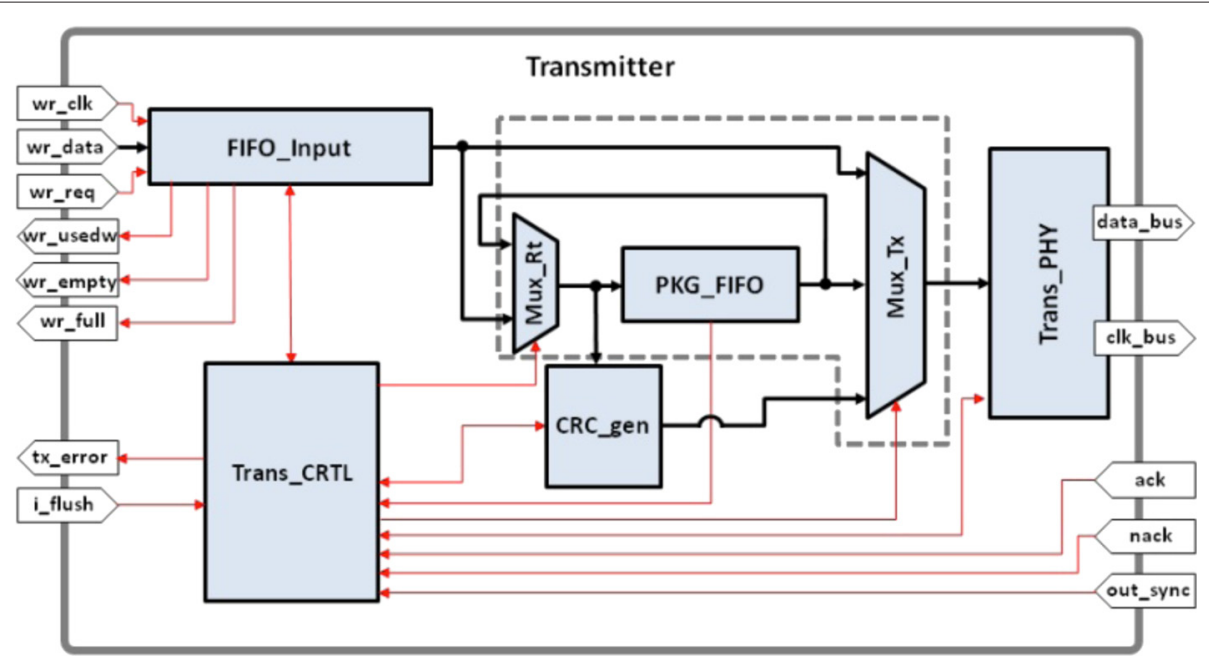

Fig. 4 Transmitter internal architecture. Block diagrams of the transmitter internal architecture

into FIFO. The $w r_{-} u s e d w$ signal indicates to the internal architecture how many words there are in FIFO. Finally, the wr_empty signal indicates when FIFO is empty and $w r$ full signal indicates when FIFO is full. All these signals have been made available so that the user can monitor the status of FIFO input and have full control of writing data for transmission.

The communication occurs on the bus through packages. By default, the size of the package to be transferred is of eight words of 32 bits, as the bus only supports this data transmission width. In this case, two words of the input FIFO (256 bits) are required to form a package. Based on this example, the transmitter removes the data from FIFO input and divides it. The first division occurs at the output of FIFO input. By default, the output is of 64 bits. The second one is made within the TRANS_PHY module that divides each data of 64 bits into two of 32 bits.

Each data package is sent to the bus, and then a checksum is immediately sent to verify the integrity of the package at the receiver. If a match is found in the checksum verification at the receiver, it sends an $A C K$ signal to the transmitter indicating that the package has been received successfully; otherwise, a $N A C K$ signal is sent and a retransmission occurs.

The retransmission circuit is highlighted in Fig. 4. This circuit is responsible for keeping the data package to be transmitted in its structure and, in case of failure, retransmit it in the fastest possible time. Because of that, this circuit has a FIFO (PKG_FIFO), which stores the package to be transmitted, and two multiplexers ( $M U X \_R T$ and $\left.M U X_{-} T X\right)$ that select the correct path for the data to be sent. The data package to be transmitted, after being required in the FIFO_INPUT, goes to the physical layer (TRANS_PHY) through the MUX_TX component. Simultaneously, a copy of the package is temporarily stored in the retransmission FIFO (PKG_FIFO) by the $M U X \_R T$ component. If there is a problem in the transmission, and the NACK signal takes a high logic level, the data previously stored in PKG_FIFO will be read and transmitted to the receiver via the $M U X_{-} T X$ and TRANS_PHY modules, respectively. A feedback loop ensures that this package can be retransmitted at other times, up to the value of MAX_RETX previously stipulated in the project. By default, this value is 10 .

The CRC generation is implemented by using a web tool called CRC tool available in [12]. This tool provides code generation CRC in both Verilog and VHDL. It allows one to choose the polynomial, the type of CRC, the data width, and the target language. The authors have chosen the polynomial $p=x^{16}+x^{15}+x^{2}+1$, default "CRC-16/USB Date" data width of 64 bits, and Verilog language.

The function generated by the tool has been adapted into the CRC_GEN module that has a state machine capable of generating the checksum of a data package. While the data package is transmitted, they will also be analyzed by CRC_GEN that calculates the CRC of the package and then immediately provides a 16-bit checksum to be transmitted over the bus. Each package corresponds to two pieces of data input into the FIFO (256 bits) that will be internally divided into eight pieces of data to transfer (32 bits each). By default, for each package, one CRC verification code is generated. It is also possible to modify the data package size to $4,8,16$, or 32 words.

Through MegaWizard, it is possible to create a CRC generator module. However, this module is proprietary, a fact which prevents its use. Thus, the CRC_GEN module has been developed with the same inputs and outputs available from Altera module, so as to maintain compatibility between versions. Figures 5 and 6 below illustrate 


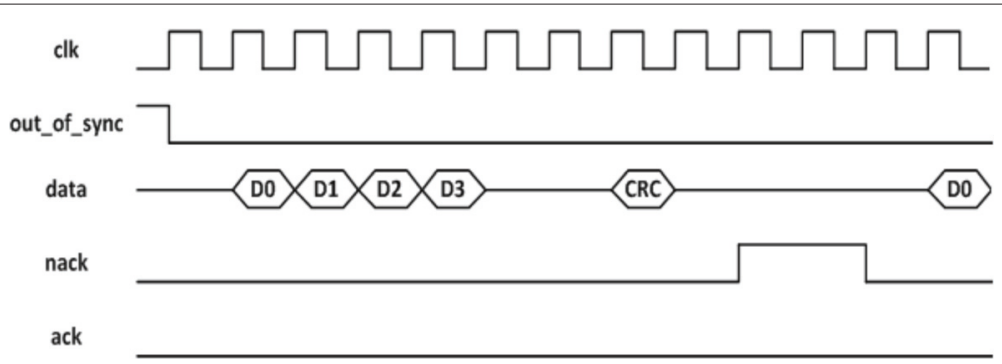

Fig. 5 Transmission failure (NACK). Waveform of an unsuccessful transmission

the communication protocol developed for the proposed architecture.

The out_of_sync signal comes from the receiver, and it is intended to inform the transmitter when its internal clock is synchronized with the $c l k \_b u s$ signal. Therefore, the transmission only occurs when the signal is at low logic level.

The TRANS_PHY module is responsible for managing and encapsulating solutions for the use of FPGA I/O pins. The use of a physical layer allows future changes in transmission patterns without major impacts on the project.

The data leaving the $M U X_{-} T X$ with 64 bits is broken into two blocks, thus forming the most significant part (data_high) and the least significant part (data_low). Soon after, the data are transmitted to the bus along with a valid signal.

The standard DDR interface was used for transmission [14]. Thus, the data is sent in both high and low clock edge, allowing greater throughput on the communication channel. Figure 7 shows TRANS_PHY internal architecture.

The ALTDDIO modules are also generated by the MegaWizard tool. They have the purpose to generate data in DDR standard, and its internal registers are implemented in silicon hard registers, i.e., directly on the FPGA microarchitecture, ensuring lower rates of skew in the data output.

The first module ALTDDIO is used with two input constants, with data_high signal connected to VCC (logic high) and data_low signal connected to GND (logic low). This configuration generates a clock output that is in phase with the data, i.e., an alignment to the rising edge of the clock related to the data valid window.

The second module ALTDDIO is used for data transmission, with its input signals (data_high and data_low) receiving a distinct portion of the data: for data_high signal, the most significant part of data (32 bits), and for data_low signal, the least significant part (32 bits).

The TRANS_CTRL module encapsulates all the necessary logic that generates the control signaling for all other modules. This logic controls the data package generation, the CRC checksum, data input through FIFO_INPUT, and retransmissions, if it is needed. It is also the responsibility of this module to indicate to the architecture, through tx_error signal, when there are any errors in data transmission. This is the case, for example, when the number of retransmissions has been achieved. Finally, the $i$ flush control signal indicates the end of data input into the FIFO. Thus, it is possible to complete the data package with zeros if necessary, maintaining the transmission pattern.

\section{Receiver}

The receiver is responsible for capturing the data bus. All signal degradation factors that interfere on the transmission channel, if any, will have their effects reflected in this module's input signals. Thus, most of the complexity in correcting these effects is located in it.

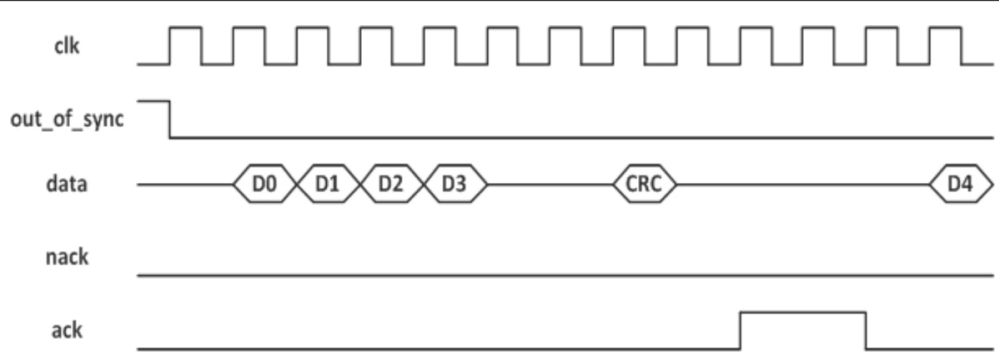

Fig. 6 Successful transmission (ACK). Waveform of a successful transmission 


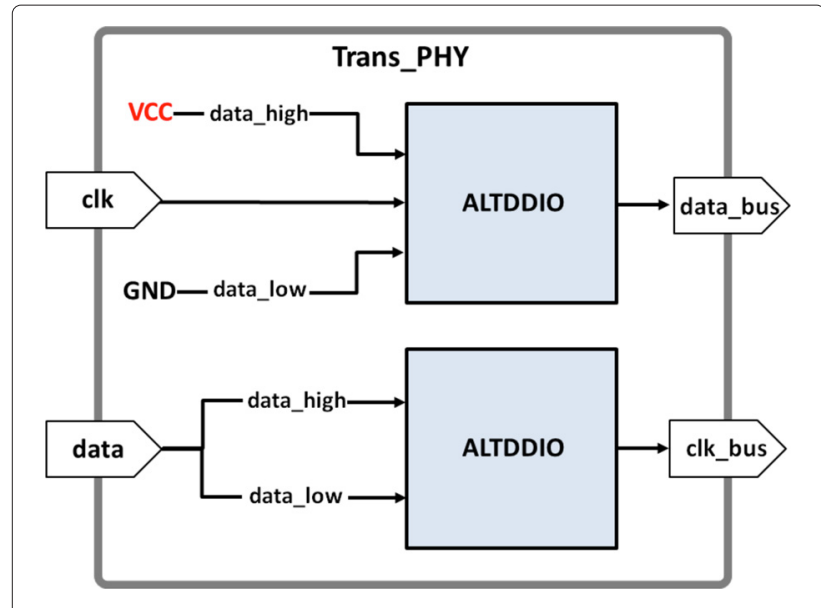

Fig. 7 TRANS_PHY architecture. Physical interface of the transmitter

The internal architecture is composed of five modules: the physical layer $\left(R E C V_{-} P H Y\right)$, two FIFOs for receiving the packages (PKG_BUFFER_1 and PKG_BUFFER_2), the CRC checker $\left(C R C_{-} C H K\right)$, the FIFO output (FIFO_OUTPUT), and the controller (RECV_CTRL) (Fig. 8).

The RECV_PHY module is responsible for receiving the transmitted data and the clock signal from the bus. The entire circuit that implements the DDR standard synchronization is also included in this module. A PLL is instantiated internally, and it is responsible for receiving the clock_bus signal and feeding the whole receiver circuit. The PLL used is generated by the MegaWizard tool and is called ALTPLL. This PLL is configured as a synchronized source. This means that it guarantees the generated clock to be fully synchronized with the communication bus clock. Figure 9 shows the RECV_PHY architecture.
The PLL circuit feeds the receiver through the data_clk signal. A locked signal is used to indicate when the PLL reaches synchronism with the input clock. Thus, the out_sync signal ensures that transactions can only occur when both the transmitter and receiver are synchronized. In order to minimize the cross-talk effects, this signal was inverted.

The circuit that is responsible for receiving the data packages comprises a demultiplexer (DEMUX_RX), two FIFOs (PKG_BUFFER_1 and PKG_BUFFER_2), and a multiplexer $\left(M U X_{-} O U T\right)$. Initially, all received data is stored in PKG_BUFFER_1. When the first package is complete, its checksum is verified, and if the package is valid, the data may follow to the FIFO output (FIFO_OUTPUT) through MUX_OUT. While this process of transferring data from the PKG_BUFFER_1 to the FIFO_OUTPUT occurs, DEMUX_RX switches and the next captured data begins to be stored in $P K G \_B U F F E R \_2$. The received package is checked, and if it is valid, the data from this buffer is also transmitted to the same FIFO_OUTPUT and the $D E M U X \_R X$ is switched again. This approach enables one to obtain a higher performance once there is no need to wait for one PKG_FIFO to be transferred to FIFO_OUTPUT to start receiving the next package. FIFOs PKG_BUFFER_1 and PKG_BUFFER_2 are of 64-bit width and 32-word depth each.

The CRC_CHK module is similar to the CRC_GEN module in the transmitter. It has the same function and is responsible for calculating the CRC value of the received packages. At the end of each package transmission, the checksum value generated by $C R C_{-} C H K$ is compared with the checksum received (generated by the CRC_GEN). In this structure, if the CRC check fails, the data is discarded before being inserted into FIFO output, and a $N A C K$ signal is sent to the transmitter requesting

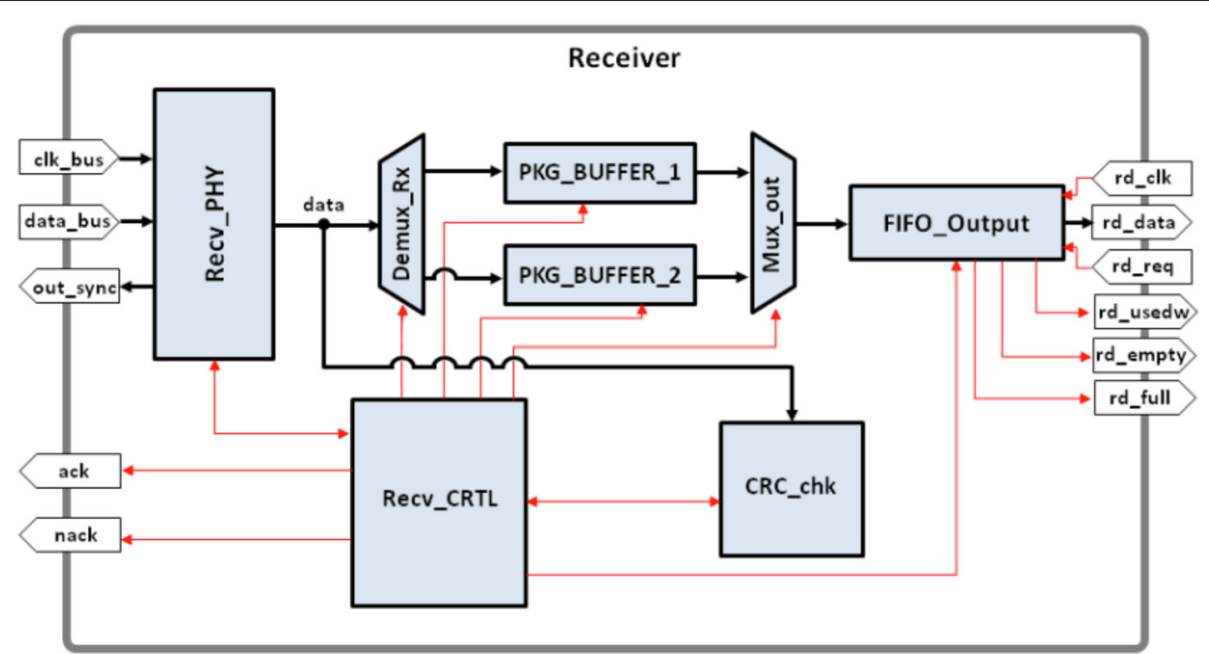

Fig. 8 Receiver internal architecture. Block diagrams of the receiver internal architecture 


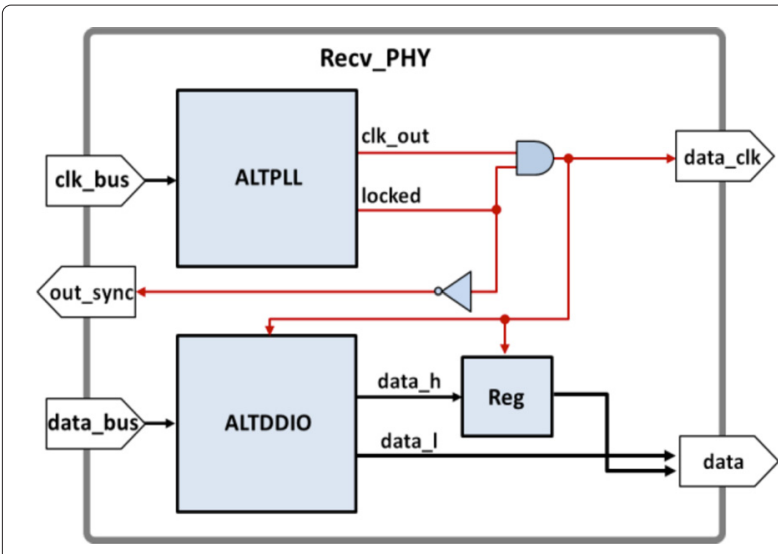

Fig. 9 RECV_PHY architecture. Physical interface of the receiver

a retransmission. If the $\mathrm{CRC}$ check is positive, the data is routed to FIFO output and an $A C K$ signal is sent to the transmitter requesting the next package.

FIFO output is exactly the same as FIFO input, located in the Transmitter. It works in two different clock domains, and it has configurable depth and width. By default, it has an input data width of 64 bits and an output data width of 128 bits with a 256-word depth. The $r d \_c l k$ signal corresponds to the clock signal architecture that synchronizes the data readings. The $r d$ req signal is used to read data from FIFO. The $r d$ _usedw signal indicates the data amount inside FIFO to the architecture. The $r d$ empty signal indicates when the FIFO is empty, and the $r d \_f u l l$ signal indicates when the FIFO is full.

The RECV_CTRL module generates the control signaling to all other modules, thus controlling data package reception, CRC verification, package transferring from the internal FIFO to the FIFO output, the internal FIFO cleaning in case of error in checksum verification, retransmission request through $N A C K$ signaling, package reception through $A C K$ signaling, and the demultiplexer $\left(D E M U X \_R X\right)$ and multiplexer $\left(M U X \_O U T\right)$ switches.

\section{Dynamic clock phase adjustment}

The data captured from the data bus must be done on the most stable moment. This occurs exactly between data transitions. The simplest way to ensure this is by establishing a phase shift in the clock bus to ensure that the clock transition occurs at the desired moment. Figure 10 illustrates that.

A fixed clock phase shift could ensure the timing of the bus clock transition to the data stability time. However, multi-platform FPGAs have particular characteristics bus width, bus length, and internal chip features - that generate different timing delays on signal propagation. Thus, it would be virtually impossible to ensure that the data was always captured at the best time. In order to

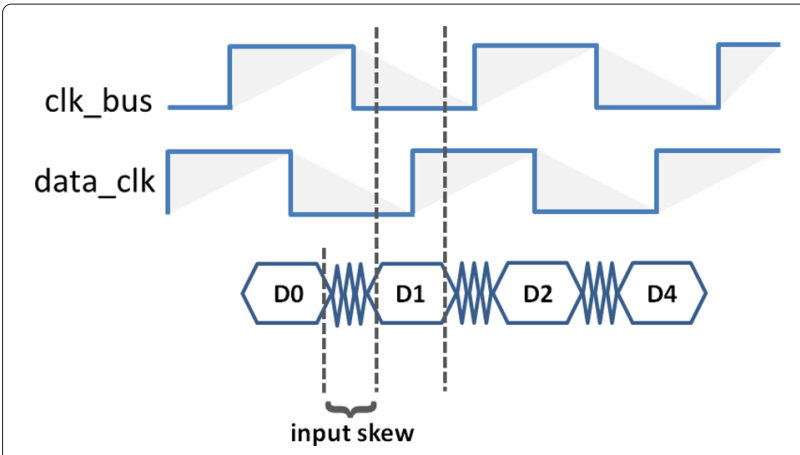

Fig. 10 Data capture in the opposite border. Data capture in the most stable moment

create a flexible communication bus for use in different platforms, a system capable of dynamically configuring and reconfiguring the clock phase when necessary was developed. The configuration is made through the PLL interface that receives the bus clock. Thus, the communication bus proposed in this work is able to self-adapt to the target platform without requiring manual configuration.

The process of identifying and dynamically adjusting the clock phase initiates after the PLL lock. After this step, the transmitter signals the receiver that the dynamic adjustment process has started by asserting high level to i_sync_start signal and starts sending a steady pattern of alternating 0's and 1's on the 32-bit data bus. The hexadecimal representation of this pattern corresponds to "0xAAAAAAAA55555555". Initially, the clock phase is set to $0^{\circ}$. The receiver compares the received data on the bus with the expected pattern. While the phase shift controller does not recognize the expected pattern, its program phase shift increments through the PLL interface. The phasestep signal is responsible for the phase increment and decrement. The phaseupdown signal determines the phase shift direction. When phaseupdown is 0 , phasestep generates an increment to the clock phase, otherwise a decrement. When the PLL has adjusted each generated pulse phase of the phasestep signal, it signals the end of this operation through the phasedone signal. When the pattern is recognized, the controller starts to count the number of increments given to the clock phase and keeps incrementing until the pattern stops to be recognized again. This counting allows the controller to get the exact size in degrees of the valid data window. The last step consists in performing decrements to the clock phase corresponding to half the number of increments performed before. This process ensures that data will be captured at exactly half of the valid data window. When this process ends, the receiver signals to the transmitter that the synchronization process has been completed through the o_sync_ready signal and begins the normal operation. If the data bus presents a configurable number 
of invalid transmissions, the dynamic adjustment of the clock phase runs again.

\section{Results}

The communication channel was tested on a GiDEL PROCStarIII platform connected to an Intel Xeon $3 \mathrm{GHz}$ computer with $4 \mathrm{~GB}$ of DDR memory through a PCIex8 bus. Different clock rates and package sizes were tested. Each test was performed considering at least $1 \mathrm{~h}$ of continuous data transfer between the FPGAs on the platform.

The data to be transferred is generated by an application at high level and sent to the host platform through the PCIex8 bus. A specific memory controller from GiDEL (GiDEL PROCMultiPort [15]) is used to transfer the data sent from the application to the transmitter. For testing purposes and correct analysis, the data received by the receiver is sent back to the host, and there, it is compared to the previously sent data.

The times presented in the result tables refer only to the effective data transmission time on the bus. The time of sending data to the host platform, the time to transfer data from the DDR2 memory (located on the platform) to the FPGA transmitter, and the time of data transmission from the receiver on the FPGA to the host were not taken into account.

As a basis for testing, the amount of transferred data during $1 \mathrm{~h}$ and a package size of 8 words was adopted. With the data amount transferred in this configuration, it was possible to repeat the tests varying the package size and measuring the transfer time.

Tables 1, 2, 3, and 4 show the results obtained for transmissions at frequencies of 50,100,120, and $150 \mathrm{MHz}$, respectively.

All the tests with the configurations presented in Tables 1, 2, 3, and 4 were successfully performed, and no transmission error was detected. For all results, a higher data rate was observed when the package size is increased from 8 to 16 words, and then from 16 to 32 words. This occurs due to the clock cycles required to generate and check the CRC at the transmitter and receiver. Once the data package becomes smaller, more cycles will be required to generate the $\mathrm{CRC}$ check, consequently increasing the overall transmission time. Figure 5 shows a transmission flow for a package size equal to 4 words. Note in the diagram that after sending the

Table 1 Results at $50 \mathrm{MHz}$

\begin{tabular}{llll}
\hline $\begin{array}{l}\text { Package } \\
\text { size }\end{array}$ & $\begin{array}{l}\text { Transfer rate } \\
(\mathrm{Gbps})\end{array}$ & $\begin{array}{l}\text { Data amount } \\
(\mathrm{Gb})\end{array}$ & $\begin{array}{l}\text { Transmission } \\
\text { time }(\mathrm{s})\end{array}$ \\
\hline 8 & 0.7 & 316.65 & 3600 \\
16 & 1.14 & 316.65 & $\sim 2200$ \\
32 & 1.68 & 316.65 & $\sim 1500$ \\
\hline
\end{tabular}

Table 2 Results at $100 \mathrm{MHz}$

\begin{tabular}{llll}
\hline $\begin{array}{l}\text { Package } \\
\text { size }\end{array}$ & $\begin{array}{l}\text { Transfer rate } \\
(\text { Gbps })\end{array}$ & $\begin{array}{l}\text { Data amount } \\
(\mathrm{Gb})\end{array}$ & $\begin{array}{l}\text { Transmission } \\
\text { time }(\mathrm{s})\end{array}$ \\
\hline 8 & 1.4 & 633.30 & 3600 \\
16 & 2.29 & 633.30 & $\sim 2200$ \\
32 & 3.37 & 633.30 & $\sim 1500$ \\
\hline
\end{tabular}

effective data, some data cycles are required to send the CRC.

According to the presented tables, it is possible to see that the data amount transferred increases with the frequency. The tests conducted at a frequency of $150 \mathrm{MHz}$ obtained the best transfer rates. For package transmissions with a size equal to 8,16 , and 32 words, transmission rates of $1.98,3.24$, and 4.76 Gbps were obtained, respectively.

It is important to mention that the same architecture developed and tested in PROCStarIII may have different transmission rates when instantiated on other platforms. Inherent characteristics to the platform design can directly influence the communication performance.

\section{Communication error test}

In order to test the package retransmission, a module that forces errors in the communication bus was implemented in the architecture. This error generator is positioned at the TRANS_PHY module output and modifies some data bits according to an insertion error rate configured in milliseconds. Thus, it is possible to simulate transmission errors and test the operation of the communication bus under these conditions. Such testing ensures the communication bus reliability in an unstable scenario.

In the presented test, one wrong bit was inserted in a period of $4 \mathrm{~ms}$. The transfers were probed at $100 \mathrm{MHz}$, and the package size was equal to eight words. The amount of transferred data was of 633.30 Gb, the same amount used in the tests at $100 \mathrm{MHz}$ and normal conditions. Table 5 presents the results.

The number of retransmissions performed in this test was of 849,871 , indicating that this was the number of packages captured in the receiver that did not match the checksum. Despite the great number of errors, the

Table 3 Results at $120 \mathrm{MHz}$

\begin{tabular}{llll}
\hline $\begin{array}{l}\text { Package } \\
\text { size }\end{array}$ & $\begin{array}{l}\text { Transfer rate } \\
\text { (Gbps) }\end{array}$ & $\begin{array}{l}\text { Data amount } \\
(\text { Gb) }\end{array}$ & $\begin{array}{l}\text { Transmission } \\
\text { time (s) }\end{array}$ \\
\hline 8 & 1.58 & 715.25 & 3600 \\
16 & 2.6 & 715.25 & $\sim 2200$ \\
32 & 3.8 & 715.25 & $\sim 1500$ \\
\hline
\end{tabular}


Table 4 Results at $150 \mathrm{MHz}$

\begin{tabular}{llll}
\hline $\begin{array}{l}\text { Package } \\
\text { size }\end{array}$ & $\begin{array}{l}\text { Transfer rate } \\
\text { (Gbps) }\end{array}$ & $\begin{array}{l}\text { Data amount } \\
(\mathrm{Gb})\end{array}$ & $\begin{array}{l}\text { Transmission } \\
\text { time }(\mathrm{s})\end{array}$ \\
\hline 8 & 1.98 & 894.06 & 3600 \\
16 & 3.24 & 894.06 & $\sim 2200$ \\
32 & 4.76 & 894.06 & $\sim 1500$ \\
\hline
\end{tabular}

retransmitted data amount was of just $25.93 \mathrm{Mb}$, causing no major impacts in relation to the total communication time. The time required for these retransmissions was approximately $144 \mathrm{~ms}$. This time was measured considering the difference between the test with and without error insertion.

After the analysis of these test results, observations showed that the communication was successful despite the errors that had been deliberately inserted in the bus. Therefore, a stable communication between the FPGAs can be obtained under similar conditions.

\section{RTM algorithm results}

The implemented communication bus was used for processing the RTM algorithm on the GiDEL PROCStarIII platform. The RTM algorithm is used in the oil and gas industry to generate images of the subsurface helping well exploration identification. This algorithm has high computational cost and therefore needs to be processed on multiple computing units, making it necessary to use four FPGAs of the GiDEL platform in this study case. Tests were performed aiming to validate the communication bus and measure the overhead generated by the communication. As a consequence, the algorithm presented the expected results and the execution time was very close to optimal, which confirms that the communication did not become the bottleneck in the algorithm execution on multiple FPGAs. After that, Table 6 presents these results. The first column lists the size of selected problems, and the following ones are the performance of a single FPGA, optimal performance with four FPGAs, and the actual measured performance, respectively. The performance unit used is of GSamples/s, which corresponds to the number of points calculated every second of execution.

\section{Discussion}

The presented implementation obtained transfer rates on the order of magnitude of Gbps. At $150 \mathrm{MHz}$, the communication bus achieved transfer rates of up to $4.76 \mathrm{Gbps}$.

Table 5 Error test at $100 \mathrm{MHz}$ clock frequency and $633.3 \mathrm{~GB}$ of data transferred

\begin{tabular}{llll}
\hline $\begin{array}{l}\text { Error insertion } \\
\text { rate (error/ms) }\end{array}$ & $\begin{array}{l}\text { Amount of } \\
\text { inserted errors }\end{array}$ & $\begin{array}{l}\text { Amount of } \\
\text { retransmitted data (Mb) }\end{array}$ & $\begin{array}{l}\text { Retransmission } \\
\text { time (ms) }\end{array}$ \\
\hline $1 / 4$ & 849,871 & 25.93 & $\sim 144$ \\
\hline
\end{tabular}

Table 6 Results of the RTM algorithm execution on GiDEL platform

\begin{tabular}{llll}
\hline $\begin{array}{l}\text { Problem } \\
\text { size }\end{array}$ & $\begin{array}{l}\text { Single FPGA } \\
\text { performance } \\
\text { (GSamples/s) }\end{array}$ & $\begin{array}{l}\text { 4 FPGAs optimal } \\
\text { performance } \\
\text { (GSamples/s) }\end{array}$ & $\begin{array}{l}4 \text { FPGAs real } \\
\text { performance } \\
\text { (GSamples/s) }\end{array}$ \\
\hline $400 \times 216 \times 216$ & 0.42 & 1.68 & 1.65 \\
$300 \times 288 \times 288$ & 0.35 & 1.42 & 1.38 \\
$1000 \times 432 \times 432$ & 0.35 & 1.42 & 1.41 \\
\hline
\end{tabular}

Although the obtained data transfer rates were satisfactory, the use of LVDS pins would certainly provide higher data transfer rates. Taking the data presented in [6] as reference, if it was possible to instantiate 32 lanes on the communication bus, each one with rates of $625 \mathrm{Mbps}$, it would be possible to obtain transfers up to $20 \mathrm{Gbps}$.

Most modern FPGAs have indicated transceivers for data transfers. Stratix V devices, from Altera, can reach transfer rates up to 28.05 Gbps [16]. However, it is essential that the PCB track routing that will accommodate these devices is done carefully, avoiding communication instabilities.

\section{Conclusions}

The use of multi-FPGA platforms enables the application of performance increase, as multiple reconfigurable devices can be used to perform the same task or work in cooperation. The use of several FPGAs integrated on a single board demands the establishment of a communication channel among such devices.

The presented work describes a bi-directional interFPGA communication bus using a DDR interface for data transmission. An architecture was designed as a reconfigurable platform, requiring only few parameter settings on the transmitter and receiver to port for different configurations.

A detection error module was also implemented. It was based on the CRC method and has also a dynamic clock phase shift adjustment in order to ensure a stable communication. The proposed architecture has been tested and validated on the GiDEL PROCStarIII platform.

The proposed communication channel achieved an excellent performance on platforms that do not have advanced features like LVDS interfaces and transceivers. The next step will be to implement it in another platform, the GiDEL PROCStarIV. This platform is based on Altera Stratix IV FPGAs and more advanced features. Thus, the authors expect to achieve higher frequencies with the use of the presented communication channel.

\section{Competing interests}

The authors declare that they have no competing interests. 


\section{Authors' contributions}

All authors have contributed to the different methodological and experimental aspects of the research. All authors read and approved the final manuscript.

\section{Acknowledgements}

The authors thank Petrobras for technical support and gratefully acknowledge the continuous support of Gidel, including the availability of prototype boards. The authors also thank FACEPE and CNPq (both Brazilian agencies) for partial financial support.

\section{Author details}

${ }^{1}$ Center for Informatics, Federal University of Pernambuco, Av. Jornalista Anibal Fernandes, s/n Cidade Universitaria, 50.740-560 Recife, Brazil. ${ }^{2}$ Statistics and Informatics Department, Federal Rural University of Pernambuco, Rua Dom Manoel de Medeiros, s/n Campus Dois Irmaos, 52.171-900 Recife, Brazil.

Received: 12 March 2014 Accepted: 9 June 2015

Published online: 17 July 2015

\section{References}

1. Hammami O, Li X, Larzul L, Burgun L (2009) Automatic design methodologies for MPSOC and prototyping on multi-FPGA platforms. In: SoC Design Conference (ISOCC), International, Busan, Korea. pp 141-146

2. Melnikova O, Hahanova I, Mostovaya K (2009) Using multi-FPGA systems for ASIC prototyping. In: CAD Systems in Microelectronics, 2009. CADSM 10th International Conference - the Experience of Designing and Application of, Lviv-Polyana, Ukraine. pp 237-239

3. Instruments T (2008) LVDS owner's manual. http://www.ti.com/lit/ml/ snla187/snla187.pdf. Accessed 11 Feb 2014

4. Tiwari A (2012) A low power high speed dual data rate acquisition system using FPGA. In: 2012 International Conference on Communication, Information Computing Technology (ICCICT), Mumbai, India. pp 1-4

5. Huixin Z, Qi H, Suhua L, Haiguang Y (2011) The design for LVDS high-speed data acquisition and transmission system based on FPGA. In: IEEE 3rd International Conference On Communication Software and Networks (ICCSN), Xi'an, China. pp 383-386

6. Xilinx Inc. http://www.xilinx.com. Accessed 11 Feb 2014

7. Altera Corporation. http://www.altera.com. Accessed 11 Feb 2014

8. Godbole P, Batth A, Ramaswamy N (2010) High speed multi-lane LVDS inter-FPGA communication link. In: IEEE International Conference OnComputational Intelligence and Computing Research (ICCIC), Coimbatore, India. pp 1-4

9. Inagi M, Takashima Y, Nakamura Y (2009) Globally optimal time-multiplexing in inter-FPGA connections for accelerating multi-FPGA systems. In: International Conference On Field Programmable Logic and Applications, Prague, Czech Republic, FPL 2009. pp 212-217

10. PARAMNet-3. http://www.cdac.in/html/htdg/products.aspx. Accessed 11 Feb 2014

11. Raptor modules. http://www.ks.cit-ec.uni-bielefeld.de/projects/raptorfamily/raptor-modules.html. Accessed 11 Feb 2014

12. GiDEL PROCStarlll. http://www.gidel.com/PROCStarlll.htm. Accessed 11 Feb 2014

13. GiDEL PROCWizard. http://www.gidel.com/procwizard.htm. Accessed 11 Feb 2014

14. Corporation L (2004) DDR interface design implementation. http://www. latticesemi.com/lit/docs/generalinfo/memory_ddr_interface_wp.pdf

15. GiDEL PROCMultiport. http://www.gidel.com/procmultiport.htm. Accessed 11 Feb 2014

16. Stratix V transceivers. http://www.altera.com/devices/fpga/stratix-fpgas/ stratix-v/transceivers/stxv-transceivers.html. Accessed 11 Feb 2014

\section{Submit your manuscript to a SpringerOpen ${ }^{\circ}$ journal and benefit from:}

- Convenient online submission

- Rigorous peer review

- Immediate publication on acceptance

- Open access: articles freely available online

- High visibility within the field

- Retaining the copyright to your article

Submit your next manuscript at $\boldsymbol{\nabla}$ springeropen.com 